\title{
SINGLE TIMESCALE STOCHASTIC APPROXIMATION FOR STOCHASTIC NASH GAMES IN COGNITIVE RADIO SYSTEMS
}

\author{
Jayash Koshal, Angelia Nedić and Uday V. Shanbhag ${ }^{\dagger}$ \\ Department of Industrial and Enterprise Systems Engineering, \\ University of Illinois, Urbana IL 61801. \\ Email: \{koshal1,angelia,udaybag\}@illinois.edu
}

\begin{abstract}
In the face of increasing demand for wireless services, the design of spectrum assignment policies has gained enormous relevance. We consider one such instance in cognitive radio systems where recent efforts have focused on the application of game-theoretic approaches. Much of this work has been restricted to deterministic regimes and this paper considers distributed schemes in the stochastic regime. The corresponding problems are seen to be stochastic Nash games over continuous strategy sets. Notably, the gradient map of player utilities is seen to be monotone mappping over the cartesian product of strategy sets, leading to a monotone stochastic variational inequality. We consider the application of projection-based stochastic approximation schemes. However, such techniques are characterized by a key shortcoming: they can accommodate strongly monotone mappings only with standard extensions of stochastic approximation schemes for merely monotone mappings is natively a two-timescale scheme. Accordingly, we consider the development of single timescale techniques for computing equilibria when the associated gradient map does not admit strong monotonicity. We develop convergence theory for distributed single-timescale stochastic approximation schemes, namely stochastic iterative proximal point method which requires exactly one projection step at every step. Finally we apply this framework to the design of cognitive radio systems in uncertain regimes under temperature-interference constraints.
\end{abstract}

Index Terms - Stochastic approximation, VI, Game theory, Cognitive Radio, Communication

\section{INTRODUCTION}

Game-theoretic models have proved to be useful in the modeling of strategic behavior in a host of problems in communication networks, including power control and flow management, amongst others. More recently, game-theoretic approaches have assumed relevance in cognitive radio (CR) systems. Such questions have become increasingly relevant in

\footnotetext{
${ }^{\dagger}$ This work has been supported by NSF CMMI 09-48905 EAGER ARRA award.
}

the face of growing demand for wireless services and the inefficiencies associated with fixed assignment policies. Cognitive radio systems represent a possible resolution by providing radio nodes with intelligence (or cognitive abilities) that allow for sensing electromagnetic variations and the ability to optimize resources. One proposal for designing spectrum sharing is through a hierarchical framework that distinguishes primary users (PUs) from secondary users (SUs); specifically, PUs are licensed spectrum holders while SUs are permitted to share the spectrum without introducing intolerable interference to PUs. The interaction of SUs can be modeled through a noncooperative game $[1,7]$ where each SU maximizes its information subject to interference constraints, given adversarial decisions. Prior work in [7] considers perfect channel state information (CSI) between primary and secondary users while imperfect CSI is assumed in [12]. In this paper we further extend this to imperfect CSI between SUs as in [5] and assume the gradient maps across players are strictly monotone. Distributed schemes for computing equilibria, such as waterfilling methods that are leveraged for obtaining best responses, have received significant attention in the recent past [7].

In this paper, we consider instances of such games where the player utilities contain expectations; the resulting class of games is a stochastic Nash game over continuous strategy sets. The associated equilibrium conditions of this game may be compactly stated as a stochastic variational inequality, for which we consider projection-based stochastic approximation algorithms. Such schemes have been recently employed for the solution of stochastic variational inequalities [4] with strongly monotone and co-coercive maps (see [3] for a definition), which to the best of our knowledge, appears to be the only existing work considering stochastic approximation methods for variational inequalities. The typical stochastic approximation procedure, first introduced by Robbins and Monro [9], works toward finding an extremum of a function $h(x)$ using the following iterative scheme:

$$
x^{k+1}=x^{k}+a_{k}\left(\nabla h\left(x^{k}\right)+M^{k+1}\right),
$$

where $M^{k+1}$ is a martingale difference sequence. Under reasonable assumptions on the stochastic errors $M^{k}$, stochastic 
approximation schemes ensure that $\left\{x^{k}\right\}$ converges almost surely to an optimal solution of the problem. Jiang and Xu [4] consider the use of stochastic approximation for the solution of stochastic variational inequalities with strongly monotone and Lipschitz continuous maps. The use of stochastic approximation methods has a long tradition in stochastic optimization, commencing with the work of Robbins and Monro [9] for differentiable problems and Ermoliev [2] for nondifferentiable problems.

This paper is motivated by the challenges associated with solving stochastic variational problems when the mappings lose strong monotonicity. In solving deterministic variational inequalities, such a departure is ably handled through techniques, such as Tikhonov regularization [11] and proximalpoint [6]. Such schemes, in general, require a solution of a regularized (well-posed) problem and an iterative process is often needed to obtain the solution. While Tikhonov regularization approach suffers from a potential drawback due to the computational aspect proximal-point method proposed by Martinet [6] (cf. [3]) and further investigated by Rockafellar [10] alleviates this problem. Proximal point methods have gained attention from various application areas communication network being one of them.

A remark is in order regarding our proposed scheme. Our goal lies in developing single timescale gradient-based distributed schemes characterized by low complexity. Such schemes lie at one end of the spectrum of best-response methods, where a gradient step is taken towards obtaining a best-response. While these techniques are not necessarily superior from the standpoint of convergence rate, they remain advantageous from the standpoint of implementability. An important question is why should players adopt this gradientbased approach, particularly if they are selfish in nature? There are two possible answers: (1) First, the game-theoretic approach can be viewed as an avenue for obtaining solutions to the multi-user centralized optimization problem allowing is to mandate the decision-making structure for each player. Second, a different viewpoint is one where we contend with equilibrium seeking in a bounded rationality setting where agents cannot take any arbitrary step; instead, they need to take "gradient steps" with an arbitrary steplength selected from a prescribed range. Note that a standard application of regularization techniques would require the solution of a sequence of strongly monotone problems with increasing exactness. Obtaining increasingly exact solutions induces significant computational burden and the need to develop alternate approaches is paramount.

The rest of the paper is organized as follows. In Section 2, we discuss a $N$-person stochastic Nash game and its associated equilibrium conditions, as given by a stochastic variational inequality. A projection-based single timescale stochastic iterative proximal point method is proposed and analyzed for this problem in Section 3 and its convergence is estabilished under an assumption of strict monotonicity of the mapping. Convergence of the resulting sequence of iterates is proved in an almost-sure sense, in a regime where players steplengths require limited coordination but are not necessarily identical across players. In Section 4, we model the competitive interactions in cognitive radio systems under temperature-interference constraints. Further, player's utilities contain expectations and the associated gradient maps are assumed to be strictly monotone. Numerical results on the performance of algorithms on one such system is presented. Finally, in Section 5, we provide some concluding remarks.

Throughout this paper, we view vectors as columns and write $x^{T}$ to denote the transpose of a vector $x$, and $x^{T} y$ to denote the inner product of vectors $x$ and $y$. We use $\|x\|$ to denote the Euclidean norm of a vector $x$, i.e., $\|x\|=\sqrt{x^{T} x}$. We use $\Pi_{X}$ to denote the Euclidean projection operator onto a set $X$, i.e., $\Pi_{X}(x) \triangleq \operatorname{argmin}_{z \in X}\|x-z\|$. The expectation of a random variable $V$ is denoted by $\mathbb{E}[V]$. Finally, we often use a.s. for almost surely.

\section{PROBLEM DESCRIPTION}

We consider an $N$-person stochastic Nash game in which the $i$ th agent solves the parameterized problem

$$
\begin{array}{ll}
\operatorname{minimize} & \mathbb{E}\left[f_{i}\left(x_{i}, x_{-i}, \xi_{i}\right)\right] \\
\text { subject to } & x_{i} \in K_{i},
\end{array}
$$

where $x_{-i}$ denotes the collection $\left\{x_{j}, j \neq i\right\}$ of decisions of all players $j$ other than player $i$. For each $i$, the variable $\xi_{i}$ is random with $\xi_{i}: \Omega_{i} \rightarrow \mathbb{R}^{n_{i}}$, and the function $\mathbb{E}\left[f_{i}\left(x_{i}, x_{-i}, \xi_{i}\right)\right]$ is convex in $x_{i}$ for all $x_{-i} \in \prod_{j \neq i} K_{j}$.

For every $i$, the set $K_{i} \subseteq \mathbb{R}^{n_{i}}$ is a closed convex set. The equilibrium conditions of the game, denoted by $\mathcal{G}$, can be characterized by scalar variational inequality problem denoted by $\mathrm{VI}(K, F)$ where

$$
F(x) \triangleq\left(\begin{array}{c}
\nabla_{x_{1}} \mathbb{E}\left[f_{1}\left(x, \xi_{1}\right)\right] \\
\vdots \\
\nabla_{x_{N}} \mathbb{E}\left[f_{N}\left(x, \xi_{N}\right)\right]
\end{array}\right), \quad K=\prod_{i=1}^{N} K_{i}
$$

with $x \triangleq\left(x_{1}, \ldots, x_{N}\right)^{T}$ and $x_{i} \in K_{i}$ for $i=1, \ldots, N$. Recall that $\operatorname{VI}(K, F)$ requires determining a vector $x^{*} \in K$ such that

$$
\left(x-x^{*}\right)^{T} F\left(x^{*}\right) \geq 0 \quad \text { for all } x \in K .
$$

We let $n=\sum_{i=1}^{N} n_{i}$, and note that the set $K$ is closed and convex set in $\mathbb{R}^{n}$, whenever the sets $K_{i}$ are closed and convex, for $i=1, \ldots, N$. The mapping $F$ is a single-valued map from $K$ to $\mathbb{R}^{n}$.

Standard deterministic algorithms for obtaining solutions to a variational inequality $\operatorname{VI}(K, F)$ require an analytical form for the gradient of the expected-value function. Yet, when the expectation is over a general measure space, such 
analytical forms are often hard to obtain. In such settings, stochastic approximation schemes assume relevance. In the remainder of this section, we describe the basic framework of stochastic approximation and the supporting convergence results.

Consider the Robbins-Monro stochastic approximation scheme for solving the stochastic variational inequality $\mathrm{VI}(K, F)$ in (2)-(3), given by

$$
x^{k+1}=\Pi_{K}\left[x^{k}-\alpha_{k}\left(F\left(x^{k}\right)+w^{k}\right)\right] \quad \text { for } k \geq 0,
$$

where $x^{0} \in K$ is an initial point, $F\left(x^{k}\right)$ is the true value of $F(x)$ at $x=x^{k}, \alpha_{k}$ is the step-size, $w^{k}=-F\left(x^{k}\right)+$ $\tilde{F}\left(x^{k}, \xi_{i}^{k}\right)$ is the stochastic error,

$$
\tilde{F}\left(x^{k}, \xi^{k}\right) \triangleq\left(\begin{array}{c}
\nabla_{x_{1}} f_{1}\left(x^{k}, \xi_{1}^{k}\right) \\
\vdots \\
\nabla_{x_{N}} f_{N}\left(x^{k}, \xi_{N}^{k}\right)
\end{array}\right) \text { and } \xi^{k} \triangleq\left(\begin{array}{c}
\xi_{1}^{k} \\
\vdots \\
\xi_{N}^{k}
\end{array}\right) \text {. }
$$

In recent work [4], the projection scheme (4) is shown to be convergent when the mapping $F$ is strongly monotone and Lipschitz continuous. In the sequel, we examine how the use of regularization methods can alleviate the need for the strong monotonicity requirement and, in fact, we show that strict monotonicity suffices.

In our analysis we use some well-known results on supermartingale convergence, which we provide for convenience. The next two results are from [8], Lemma 10, page 49.

Lemma 1 (Lemma 10, Pg. 49 [8]). Let $V_{k}$ be a sequence of non-negative random variables adapted to $\sigma$-algebra $\mathcal{F}_{k}$ and such that almost surely

$$
\mathbb{E}\left[V_{k+1} \mid \mathcal{F}_{k}\right] \leq\left(1-u_{k}\right) V_{k}+\beta_{k} \quad \text { for all } k \geq 0,
$$

where $0 \leq u_{k} \leq 1, \beta_{k} \geq 0$, and

$$
\sum_{k=0}^{\infty} u_{k}=\infty, \quad \sum_{k=0}^{\infty} \beta_{k}<\infty, \quad \frac{\beta_{k}}{u_{k}} \rightarrow 0 .
$$

Then, $V_{k} \rightarrow 0$ a.s.

Lemma 2 (Lemma 11, Pg. 50 [8]). Let $V_{k}, u_{k}, \beta_{k}$ and $\gamma_{k}$ be non-negative random variables adapted to $\mathcal{F}_{k}$. If almost surely $\sum_{k=1}^{\infty} u_{k}<\infty, \sum_{k=1}^{\infty} \beta_{k}<\infty$, and

$$
\mathbb{E}\left[V_{k+1} \mid \mathcal{F}_{k}\right] \leq\left(1+u_{k}\right) V_{k}-\gamma_{k}+\beta_{k} \quad \text { for all } k \geq 0,
$$

then $\left\{V_{k}\right\}$ is convergent a.s. and $\sum_{k=1}^{\infty} \gamma_{k}<\infty$.

Throughout the rest of the paper, we use $\mathcal{F}_{k}$ to denote the sigma-field generated by the initial point $x^{0}$ and errors $w^{\ell}$ for $\ell=0,1, \ldots, k$, i.e.,

$$
\mathcal{F}_{k}=\left\{x^{0},\left(w^{\ell}, \ell=0,1, \ldots, k\right)\right\} \quad \text { for } k \geq 0 .
$$

Now, we specify our assumptions for $\operatorname{VI}(K, F)$ in (2)-(3) and the algorithm (4).
Assumption 1. Let the following hold:

(a) The sets $K_{i} \subseteq \mathbb{R}^{n_{i}}$ are closed and convex;

(b) The mapping $F: K \rightarrow \mathbb{R}^{n}$ is strictly monotone and Lipschitz, with constant $L$ over the set $K$;

(c) The stochastic error is such that $\mathbb{E}\left[w^{k} \mid \mathcal{F}_{k}\right]=0$ and $\sum_{k=1}^{\infty} \alpha_{k}^{2} \mathbb{E}\left[\left\|w^{k}\right\|^{2} \mid \mathcal{F}_{k}\right]<\infty$ a.s.

Since the map $F$ is strictly monotone, the solution to $\operatorname{VI}(K, F)$ is unique whenever it exists (see 2.3.3 Theorem in [3]). Note, however, that the strict monotonicity of $F$ is not enough to guarantee the existence of a solution when the set $K$ is closed and convex.

We note that if Assumption 1(b) is strengthened to $F$ being strongly monotone and the stepsize $\alpha_{k}$ is such that $\sum_{k=1}^{\infty} \alpha_{k}=\infty$, then we immediately have almost sure convergence of the algorithm, which has been established in [4].

\section{STOCHASTIC ITERATIVE PROXIMAL-POINT SCHEMES}

Proximal-point methods, a class of techniques that appear to have been first studied by Martinet [6], and subsequently by Rockafellar [10] has a long history for addressing monotone variational inequalities. A more recent description in the context of maximal-monotone operators can be found in [3]. We begin with a description of such methods in the context of a monotone variational inequality $\operatorname{VI}(K, F)$ where $F$ is a continuous and monotone mapping. Recall that Tikhonov-based techniques require the construction of a sequence $x_{k}$, where $x_{k}$ is the unique solution of $\operatorname{VI}\left(K, F+\epsilon_{k} \mathbf{I}\right)$ and $\epsilon_{k}>0$. Under suitable assumptions and given a positive sequence $\left\{\epsilon^{k}\right\}$ with $\epsilon_{k} \rightarrow 0$, we have that $\lim _{k \rightarrow \infty} x_{k}=x^{*}$, where $x^{*}$ is some solution of $\operatorname{VI}(K, F)$. In proximal-point methods, the convergence to a single solution of $\mathrm{VI}(K, F)$ is obtained through the addition of a proximal term $\theta\left(x_{k}-x_{k-1}\right)$, where $\theta$ is a fixed positive parameter. In effect, $x_{k}=\operatorname{SOL}(K, F+$ $\left.\theta\left(\mathbf{I}-x_{k-1}\right)\right)$ and convergence may be guaranteed under suitable assumptions.

When employing a proximal-point method for solving the $\mathrm{VI}(K, F)$ associated with problem (1), a crucial shortcoming of standard proximal-point schemes lies in the need to solve a sequence of variational problems, a natively two-timescale scheme. Accordingly we develop a single time-scale stochastic iterative proximal point method (IPP) in which the centering term $x_{k-1}$ is updated after every projection step rather than when obtains an accurate solution of $\mathrm{VI}(K, F+\theta(\mathbf{I}-$ $\left.x_{k-1}\right)$ ). The distributed stochastic form of the proximal-point scheme is given by

$$
x_{i}^{k+1}=\Pi_{K_{i}}\left[x_{i}^{k}-\alpha_{k, i}\left(F_{i}\left(x^{k}\right)+\theta_{k}\left(x_{i}^{k}-x_{i}^{k-1}\right)+w_{i}^{k}\right)\right],
$$

where $\alpha_{k, i}$ is the stepsize chosen by the $i$ th user at the $k$ th iterate, $\theta_{k}>0$ is the prox-parameter. 
Before providing a detailed analysis of the convergence properties of this scheme, we examine the relationship between the proposed iterative proximal point method and the standard gradient projection method. An iterative proximalpoint scheme for $\operatorname{VI}(K, F)$ necessitates an update given by

$$
\begin{aligned}
x_{k+1} & =\Pi_{K}\left[x_{k}-\gamma_{k}\left(F\left(x_{k}\right)+\theta\left(x_{k}-x_{k-1}\right)\right)\right] \\
& =\Pi_{K}\left[\left(\left(1-\gamma_{k} \theta\right) x_{k}+\gamma_{k} \theta x_{k-1}\right)-\gamma_{k} F\left(x_{k}\right)\right] \\
& =\Pi_{K}\left[x_{k}(\theta)-\gamma_{k} F\left(x_{k}\right)\right],
\end{aligned}
$$

where $x_{k}(\theta) \triangleq\left(1-\gamma_{k} \theta\right) x_{k}+\gamma_{k} \theta x_{k-1}$. Therefore, when $\theta \equiv 0$, the method reduces to the standard gradient projection scheme. More generally, one can view the proximal-point method as employing a convex combination of the old iterate $x_{k-1}$ and $x_{k}$ instead of $x_{k}$ in the standard gradient scheme. Furthermore, as $\gamma_{k} \rightarrow 0$, the update rule starts resembling the standard gradient scheme more closely. In our discussion, we allow $\theta$ to vary at every iteration; in effect, we employ a sequence $\theta_{k}$ which can grow to $+\infty$ but at a sufficiently slow rate.

We now examine the global convergence of the distributed stochastic proximal-point scheme given by (5) obtained for a strictly monotone $F$. We impose the following conditions on the stepsizes and the prox-parameter sequence $\left\{\theta_{k}\right\}$.

Assumption 2. Let $\alpha_{k, \max }=\max _{1 \leq i \leq N}\left\{\alpha_{k, i}\right\}, \alpha_{k, \min }=$ $\min _{1 \leq i \leq N}\left\{\alpha_{k, i}\right\}$ and the following hold:

(a) $\alpha_{k, \max } \theta_{k} \leq\left(1+2 \alpha_{k, \max }^{2} L^{2}\right) \alpha_{k-1, \min } \theta_{k-1}$ for all $k \geq$ 1 , and

$$
\lim _{k \rightarrow \infty} \frac{\alpha_{k, \max }^{2} \theta_{k}}{\alpha_{k, \min }}=c \text { with } c \in[0,1 / 2) ;
$$

(b) $\sum_{k=0}^{\infty} \alpha_{k, i}=\infty$ and $\sum_{k=0}^{\infty} \alpha_{k, i}^{2}<\infty$ for all $i$;

(c) $\sum_{k=0}^{\infty}\left(\alpha_{k, \max }-\alpha_{k, \min }\right)<\infty$.

In the next proposition, using Assumption 2 we show almost sure convergence of the method. Immediately after this result, we provide an example for the stepsizes and prox parameters satisfying Assumption 2.

Proposition 1. Let Assumption 1 hold and assume that the $\operatorname{VI}(K, F)$ has a solution. Also, let the steplengths and the prox-parameters satisfy Assumption 2. Then, the sequence $\left\{x^{k}\right\}$ generated by method (5) converges almost surely to the solution $x^{*}$ of $\operatorname{VI}(K, F)$.

Proof. By using $x_{i}^{*}=\Pi_{K_{i}}\left[x_{i}^{*}-\alpha_{k, i} F_{i}\left(x^{*}\right)\right]$ and the nonexpansivity of the Euclidean projection operator we observe that $\left\|x_{i}^{k+1}-x_{i}^{*}\right\|$ can be expressed as follows

$$
\begin{aligned}
& \left\|x_{i}^{k+1}-x_{i}^{*}\right\|^{2} \\
& =\| \Pi_{K_{i}}\left[x_{i}^{k}-\alpha_{k, i}\left(F_{i}\left(x^{k}\right)+\theta_{k}\left(x_{i}^{k}-x_{i}^{k-1}\right)+w_{i}^{k}\right)\right] \\
& -\Pi_{K_{i}}\left[x_{i}^{*}-\alpha_{k, i} F_{i}\left(x^{*}\right)\right] \|^{2} \\
& \leq \|\left(x_{i}^{k}-x_{i}^{*}\right)-\alpha_{k, i}\left(F_{i}\left(x^{k}\right)-F_{i}\left(x^{*}\right)\right) \\
& \alpha_{k, i}\left(\theta_{k}\left(x_{i}^{k}-x_{i}^{k-1}\right)+w_{i}^{k}\right) \|^{2} .
\end{aligned}
$$

Further, the right hand side of preceding relation can be expanded as

$$
\begin{aligned}
\mathrm{RHS} & =\left\|x_{i}^{k}-x_{i}^{*}\right\|^{2}+\alpha_{k, i}^{2}\left\|F_{i}\left(x^{k}\right)-F_{i}\left(x^{*}\right)\right\|^{2}+\alpha_{k, i}^{2}\left\|w_{i}^{k}\right\|^{2} \\
& +\left(\alpha_{k, i} \theta_{k}\right)^{2}\left\|x_{i}^{k}-x_{i}^{k-1}\right\|^{2}-2 \alpha_{k, i}\left(x_{i}^{k}-x_{i}^{*}\right)^{T}\left(F_{i}\left(x^{k}\right)-F_{i}\left(x^{*}\right)\right) \\
& -2 \alpha_{k, i} \theta_{k}\left(x_{i}^{k}-x_{i}^{*}\right)^{T}\left(x_{i}^{k}-x_{i}^{k-1}\right)-2 \alpha_{k, i}\left(x_{i}^{k}-x_{i}^{*}\right)^{T} w_{i}^{k} \\
& +2 \alpha_{k, i}^{2} \theta_{k}\left(F_{i}\left(x^{k}\right)-F_{i}\left(x^{*}\right)\right)^{T}\left(x_{i}^{k}-x_{i}^{k-1}\right) \\
& +2 \alpha_{k, i}^{2}\left(F_{i}\left(x^{k}\right)-F_{i}\left(x^{*}\right)\right)^{T} w_{i}^{k}+2 \alpha_{k, i}^{2} \theta_{k}\left(x_{i}^{k}-x_{i}^{k-1}\right)^{T} w_{i}^{k} .
\end{aligned}
$$

Taking expectation and using $\mathbb{E}\left[w_{i}^{k} \mid \mathcal{F}_{k}\right]=0$ (Assumption 1(c)), we obtain

$$
\begin{aligned}
& \mathbb{E}\left[\left\|x_{i}^{k+1}-x_{i}^{*}\right\|^{2} \mid \mathcal{F}_{k}\right] \\
& \leq\left\|x_{i}^{k}-x_{i}^{*}\right\|^{2}+\alpha_{k, i}^{2}\left\|F_{i}\left(x^{k}\right)-F_{i}\left(x^{*}\right)\right\|^{2}+\alpha_{k, i}^{2}\left\|w_{i}^{k}\right\|^{2} \\
& +\left(\alpha_{k, i} \theta_{k}\right)^{2}\left\|x_{i}^{k}-x_{i}^{k-1}\right\|^{2}-2 \alpha_{k, i} \theta_{k}\left(x_{i}^{k}-x_{i}^{*}\right)^{T}\left(x_{i}^{k}-x_{i}^{k-1}\right) \\
& -2 \alpha_{k, i}\left(x_{i}^{k}-x_{i}^{*}\right)^{T}\left(F_{i}\left(x^{k}\right)-F_{i}\left(x^{*}\right)\right) \\
& +2 \alpha_{k, i}^{2} \theta_{k}\left(F_{i}\left(x^{k}\right)-F_{i}\left(x^{*}\right)\right)^{T}\left(x_{i}^{k}-x_{i}^{k-1}\right)
\end{aligned}
$$

Let $\alpha_{k, \max }=\max _{1 \leq i \leq N}\left\{\alpha_{k, i}\right\}$ and $\alpha_{k, \min }=\min _{1 \leq i \leq N}\left\{\alpha_{k, i}\right\}$. Summing over all $i$ and using Lipschitz continuity of $F$ (Assumption 1(b)) we arrive at

$$
\begin{aligned}
& \mathbb{E}\left[\left\|x^{k+1}-x^{*}\right\|^{2} \mid \mathcal{F}_{k}\right] \\
& \leq\left(1+\alpha_{k, \max }^{2} L^{2}\right)\left\|x^{k}-x^{*}\right\|^{2}+\alpha_{k, \max }^{2} \mathbb{E}\left[\left\|w^{k}\right\|^{2} \mid \mathcal{F}_{k}\right] \\
& +\left(\alpha_{k, \max } \theta_{k}\right)^{2}\left\|x^{k}-x^{k-1}\right\|^{2} \\
& -\underbrace{2 \sum_{i=1}^{N} \alpha_{k, i}\left(x_{i}^{k}-x_{i}^{*}\right)^{T}\left(F_{i}\left(x^{k}\right)-F_{i}\left(x^{*}\right)\right)}_{\text {Term 1 }} \\
& -\underbrace{2 \sum_{i=1}^{N} \alpha_{k, i} \theta_{k}\left(x_{i}^{k}-x_{i}^{*}\right)^{T}\left(x_{i}^{k}-x_{i}^{k-1}\right)}_{\text {Term 2 }} \\
& -\underbrace{2 \sum_{i=1}^{N} \alpha_{k, i}^{2} \theta_{k}\left(F_{i}\left(x^{k}\right)-F_{i}\left(x^{*}\right)\right)^{T}\left(x_{i}^{k}-x_{i}^{k-1}\right)}_{\text {Term 3 }} .
\end{aligned}
$$

By adding and subtracting $2 \alpha_{k, \min }\left(x_{i}^{k}-x_{i}^{*}\right)^{T}\left(F_{i}\left(x^{k}\right)-\right.$ 
$\left.F_{i}\left(x^{*}\right)\right)$ to the each term of Term 1 we see that

$$
\begin{aligned}
\text { Term } 1 & =-2 \alpha_{k, \min } \sum_{i=1}^{N}\left(x_{i}^{k}-x_{i}^{*}\right)^{T}\left(F_{i}\left(x^{k}\right)-F_{i}\left(x^{*}\right)\right) \\
& -2 \sum_{i=1}^{N}\left(\alpha_{k, i}-\alpha_{k, \min }\right)\left(x_{i}^{k}-x_{i}^{*}\right)^{T}\left(F_{i}\left(x^{k}\right)-F_{i}\left(x^{*}\right)\right) \\
& \leq-2 \alpha_{k, \min }\left(x^{k}-x^{*}\right)^{T}\left(F\left(x^{k}\right)-F\left(x^{*}\right)\right) \\
& +2\left(\alpha_{k, \max }-\alpha_{k, \min }\right) \sum_{i=1}^{N}\left\|x_{i}^{k}-x_{i}^{*}\right\|\left\|F\left(x^{k}\right)-F\left(x^{*}\right)\right\| \\
& \leq-2 \alpha_{k, \min }\left(x^{k}-x^{*}\right)^{T}\left(F\left(x^{k}\right)-F\left(x^{*}\right)\right) \\
& +2\left(\alpha_{k, \max }-\alpha_{k, \min }\right)\left\|x^{k}-x^{*}\right\|\left\|F\left(x^{k}\right)-F\left(x^{*}\right)\right\| \\
& \leq-2 \alpha_{k, \min }\left(x^{k}-x^{*}\right)^{T}\left(F\left(x^{k}\right)-F\left(x^{*}\right)\right) \\
& +2\left(\alpha_{k, \max }-\alpha_{k, \min }\right) L\left\|x^{k}-x^{*}\right\|^{2},
\end{aligned}
$$

by Hölder's inequality and Lipschitz continuity of $F$.

We next estimate Term 2. Since $2(x-y)^{T}(x-z)=$ $\|x-y\|^{2}+\|x-z\|^{2}-\|y-z\|^{2}$, it follows that

\section{Term 2}

$$
\begin{aligned}
& =-\sum_{i=1}^{N} \alpha_{k, i} \theta_{k}\left[\left\|x_{i}^{k}-x_{i}^{*}\right\|^{2}+\left\|x_{i}^{k}-x_{i}^{k-1}\right\|^{2}-\left\|x_{i}^{k-1}-x_{i}^{*}\right\|^{2}\right] \\
& \leq-\alpha_{k, \min } \theta_{k} \sum_{i=1}^{N}\left[\left\|x_{i}^{k}-x_{i}^{*}\right\|^{2}+\left\|x_{i}^{k}-x_{i}^{k-1}\right\|^{2}\right] \\
& +\alpha_{k, \max } \theta \sum_{i=1}^{N}\left\|x_{i}^{k-1}-x_{i}^{*}\right\|^{2} \\
& =-\alpha_{k, \min } \theta_{k}\left[\left\|x^{k}-x^{*}\right\|^{2}+\left\|x^{k}-x^{k-1}\right\|^{2}\right] \\
& +\alpha_{k, \max } \theta_{k}\left\|x^{k-1}-x^{*}\right\|^{2} .
\end{aligned}
$$

We now consider Term 3. Using $2 x^{T} y \leq\|x\|^{2}+\|y\|^{2}$ and Lipschitz continuity of $F$, we obtain

$$
\begin{aligned}
\text { Term } 3 & \leq \sum_{i=1}^{N} \alpha_{k, i}^{2}\left(\left\|F_{i}\left(x^{k}\right)-F_{i}\left(x^{*}\right)\right\|^{2}+\theta_{k}^{2}\left\|x_{i}^{k}-x_{i}^{k-1}\right\|^{2}\right) \\
& \leq \alpha_{k, \max }^{2} \sum_{i=1}^{N}\left(\left\|F_{i}\left(x^{k}\right)-F_{i}\left(x^{*}\right)\right\|^{2}+\theta_{k}^{2}\left\|x_{i}^{k}-x_{i}^{k-1}\right\|^{2}\right) \\
& \leq \alpha_{k, \max }^{2}\left(\left\|F\left(x^{k}\right)-F\left(x^{*}\right)\right\|^{2}+\theta_{k}^{2}\left\|x^{k}-x^{k-1}\right\|^{2}\right) \\
& \leq \alpha_{k, \max }^{2}\left(L^{2}\left\|x^{k}-x^{*}\right\|^{2}+\theta_{k}^{2}\left\|x^{k}-x^{k-1}\right\|^{2}\right) .
\end{aligned}
$$

Combining (7) with (9), (10) and (11) we obtain

$$
\begin{aligned}
& \mathbb{E}\left[\left\|x^{k+1}-x^{*}\right\|^{2} \mid \mathcal{F}_{k}\right] \\
& \leq\left(1+2 \alpha_{k, \max }^{2} L^{2}+2\left(\alpha_{k, \max }-\alpha_{k, \min }\right) L\right)\left\|x^{k}-x^{*}\right\|^{2} \\
& +\alpha_{k, \max } \theta_{k}\left\|x^{k-1}-x^{*}\right\|^{2}-\alpha_{k, \min } \theta_{k}\left\|x^{k}-x^{*}\right\|^{2} \\
& -\alpha_{k, \min } \theta_{k}\left(1-\frac{2 \alpha_{k, \text { max }}^{2} \theta_{k}}{\alpha_{k, \min }}\right)\left\|x^{k}-x^{k-1}\right\|^{2} \\
& -2 \alpha_{k, \min }\left(x^{k}-x^{*}\right)^{T}\left(F\left(x^{k}\right)-F\left(x^{*}\right)\right) \\
& +\alpha_{k, \max }^{2} \mathbb{E}\left[\left\|w^{k}\right\|^{2} \mid \mathcal{F}_{k}\right],
\end{aligned}
$$

By Assumption 2(a) we have

$$
\begin{aligned}
\alpha_{k, \max } \theta_{k} & \leq\left(1+2 \alpha_{k, \text { max }}^{2} L^{2}\right) \alpha_{k-1, \min } \theta_{k-1} \\
& \leq\left(1+2 \alpha_{k, \text { max }}^{2} L^{2}+2\left(\alpha_{k, \max }-\alpha_{k, \min }\right) L\right) \alpha_{k-1, \min } \theta_{k-1} .
\end{aligned}
$$

Using this, moving the term $-\alpha_{k, \min } \theta_{k}\left\|x^{k}-x^{*}\right\|^{2}$ on the other side of inequality (12), and noting that

$$
\frac{2 \alpha_{k, \max }^{2} \theta_{k}}{\alpha_{k, \min }} \leq d \quad \text { for some } d \in(0,1) \text { and for } k \geq K,
$$

with sufficiently large $K$ (since $\frac{2 \alpha_{k, \max }^{2} \theta_{k}}{\alpha_{k, \min }} \rightarrow 2 c$ with $2 c<1$ by Assumption 2(a)), we further see that for $k \geq K$,

$$
\begin{aligned}
& \mathbb{E}\left[\left\|x^{k+1}-x^{*}\right\|^{2} \mid \mathcal{F}_{k}\right]+\alpha_{k, \min } \theta_{k}\left\|x^{k}-x^{*}\right\|^{2} \\
& \leq\left(1+2 \alpha_{k, \max }^{2} L^{2}+2\left(\alpha_{k, \max }-\alpha_{k, \min }\right) L\right) \\
& \left(\left\|x^{k}-x^{*}\right\|^{2}+\alpha_{k-1, \min } \theta_{k-1}\left\|x^{k-1}-x^{*}\right\|^{2}\right) \\
& -\alpha_{k, \min } \theta_{k}(1-d)\left\|x^{k}-x^{k-1}\right\|^{2}+\alpha_{k, \max }^{2} \mathbb{E}\left[\left\|w^{k}\right\|^{2} \mid \mathcal{F}_{k}\right] \\
& -2 \alpha_{k, \min }\left(x^{k}-x^{*}\right)^{T}\left(F\left(x^{k}\right)-F\left(x^{*}\right)\right) .
\end{aligned}
$$

Thus, according to Lemma 2 (that holds for all $k$ large enough) we have for the solution $x^{*}$,

$$
\begin{gathered}
\left\{\left\|x^{k}-x^{*}\right\|^{2}+\alpha_{k-1} \theta_{k-1}\left\|x^{k-1}-x^{*}\right\|^{2}\right\} \text { converges a.s. } \\
\sum_{k=0}^{\infty} \alpha_{k}\left(x^{k}-x^{*}\right)^{T}\left(F\left(x^{k}\right)-F\left(x^{*}\right)\right)<\infty \text { a.s. }
\end{gathered}
$$

Relation (14) implies that the sequence $\left\{x^{k}\right\}$ is almost surely bounded, so it has accumulation points almost surely. Since $K$ is closed and $\left\{x^{k}\right\} \subset K$, it follows that all the accumulation points of $\left\{x^{k}\right\}$ belong to $K$. By (15) and the condition $\sum_{k=0}^{\infty} \alpha_{k}=\infty$ (Assumption 2(b)) it follows that $\left(x^{k}-x^{*}\right)^{T}\left(F\left(x^{k}\right)-F\left(x^{*}\right)\right) \rightarrow 0$ along a subsequence almost surely. This and strict monotonicity imply that $\left\{x^{k}\right\}$ has one accumulation point, say $\tilde{x}$, that must coincide with the solution $x^{*}$. By relation (14) it follows that the whole sequence must converge to the random point $\tilde{x}$ almost surely.

It is worth mentioning that there exist stepsize sequences $\left\{\alpha_{k, i}\right\}$ and centering term sequence $\left\{\theta_{k}\right\}$ such that the conditions of Assumption 2 are satisfied. Here, we discuss how one may go about selecting such sequences. Consider user $i$ stepsize $\alpha_{k, i}$ of the the form $\alpha_{k, i}=\left(k+\eta_{i}\right)^{-a}$ for some $a \in(1 / 2,1]$ and a random $\eta_{i}$ with uniform distribution over an interval $[-\eta, \eta]$ for some $\eta>0$. Let $\theta_{k}=\frac{\alpha_{k, \min }}{\alpha_{k, \max }} \theta_{k-1}$. Then, the conditions of Assumption 2 are satisfied. (the verification can be omitted due to space requirement) To see this, we first show the limit condition in Assumption 2(a). We have

$$
\lim _{k \rightarrow \infty} \frac{\alpha_{k, \max }}{\alpha_{k, \min }} \alpha_{k, \max } \theta_{k}=\lim _{k \rightarrow \infty} \alpha_{k, \max } \theta_{k-1} .
$$

Since $0<\frac{\alpha_{k, \min }}{\alpha_{k, \max }} \leq 1$ for all $k$, from the form of $\theta_{k}$ we see that

$$
\lim _{k \rightarrow \infty} \theta_{k}=\lim _{k \rightarrow \infty} \alpha_{k, \max } \prod_{j=1}^{k-1} \frac{\alpha_{j, \min }}{\alpha_{j, \max }} \theta_{0} \leq \lim _{k \rightarrow \infty} \alpha_{k, \max } \theta_{0} .
$$


Since $\lim _{k \rightarrow \infty} \alpha_{k, \max }=0$, it follows that $\lim _{k \rightarrow \infty} \theta_{k}=0$. Therefore

$$
\lim _{k \rightarrow \infty} \frac{\alpha_{k, \max }}{\alpha_{k, \min }} \alpha_{k, \max } \theta_{k}=\lim _{k \rightarrow \infty} \alpha_{k, \max } \lim _{k \rightarrow \infty} \theta_{k-1}=0 .
$$

The conditions of Assumption 2(b) hold trivially for $a \in$ $(1 / 2,1]$. Also, if we let $\eta_{\max }=\max _{1 \leq i \leq N}\left\{\eta_{i}\right\}$ and $\eta_{\min }=$ $\min _{1 \leq i \leq N}\left\{\eta_{i}\right\}$, then for the condition of Assumption 2(c), we have

$$
\begin{aligned}
& \alpha_{k, \text { max }}-\alpha_{k, \min } \\
& =\left(k+\eta_{\min }\right)^{-a}-\left(k+\eta_{\max }\right)^{-a} \\
& =\left(k+\eta_{\max }\right)^{-a}\left(\frac{\left(k+\eta_{\min }\right)^{-a}}{\left(k+\eta_{\max }\right)^{-a}}-1\right) \\
& =\left(k+\eta_{\max }\right)^{-a}\left(\left(1-\frac{\eta_{\max }-\eta_{\min }}{k+\eta_{\max }}\right)^{-a}-1\right) \\
& \approx\left(k+\eta_{\max }\right)^{-a}\left(1+a \frac{\eta_{\max }-\eta_{\min }}{k+\eta_{\max }}+O\left(1 / k^{2}\right)-1\right)=O\left(1 / k^{1+a}\right),
\end{aligned}
$$

which is summable for $a \geq 0$. It remains to verify the relation in Assumption 2(a). For this, we note that

$$
\begin{aligned}
\frac{\left(1+2 \alpha_{k, \text { max }}^{2} L^{2}\right) \alpha_{k-1, \min } \theta_{k-1}}{\alpha_{k, \max } \theta_{k}} & \geq \frac{\alpha_{k-1, \min } \theta_{k-1}}{\alpha_{k, \max } \theta_{k}} \\
& \geq \frac{\alpha_{k, \min } \theta_{k-1}}{\alpha_{k, \max } \theta_{k}}=1
\end{aligned}
$$

were we use the decreasing nature of $\alpha_{k, \min }$ to conclude $\alpha_{k-1, \min } \theta_{k-1} \geq \alpha_{k, \min } \theta_{k-1}$. Therefore an acceptable choice of $\theta_{k}$ is given by $\theta_{k}=\frac{\alpha_{k, \min } \theta_{k-1}}{\alpha_{k, \max }}$ and for such a choice all the conditions of Assumption 2 are satisfied.

For the above given user stepsize choice, another possible choice for prox parameter $\theta_{k}$ is $\theta_{k}=\theta$ where $\theta$ is a positive constant. In this case, it can be seen that Assumption 2 holds for $\eta \in[0,1 / 2]$. We verify the relation in Assumption 2(a) for completeness. We do this by showing the non-negativity of the expression $C=\left(1+2 \alpha_{k, \max }^{2} L^{2}\right) \alpha_{k-1, \min } \theta-\alpha_{k, \max } \theta$ for $\eta \in[0,1 / 2]$. For this, by the form of the users stepsizes, we have

$C=\theta\left(\left(1+2 \alpha_{k, \max }^{2} L^{2}\right)\left(k-1+\eta_{\max }\right)^{-a}-\left(k+\eta_{\min }\right)^{-a}\right)$.

Through the use of the expansion $(1+1 / k)^{n} \approx 1+n / k+$ $O\left(1 / k^{2}\right)$, we further have

$$
\begin{aligned}
C & \approx \theta k^{-a}\left(\left(1+2 \alpha_{k, \max }^{2} L^{2}\right)\left(1+a \frac{1-\eta_{\max }}{k}\right)-\left(1-a \frac{\eta_{\min }}{k}\right)\right) \\
& \geq \theta k^{-a}\left(1+2 \alpha_{k, \max }^{2} L^{2}+a \frac{1-\eta_{\max }}{k}-1+a \frac{\eta_{\min }}{k}\right) \\
& \geq \theta k^{-a}\left(2 \alpha_{k, \max }^{2} L^{2}+a \frac{1-2 \eta}{k}\right) \\
& \geq 0
\end{aligned}
$$

where we use $\eta_{\max } \leq \eta, \eta_{\min } \geq-\eta$ and $\eta \in[0,1 / 2]$.

\section{COGNITIVE RADIO SYSTEMS}

In this section, we consider the application of the proposed scheme to the design of cognitive radio systems. Gametheoretic approaches in such regimes were developed in [7] and extended to stochastic settings in [13]. The use of cognitive radio (CR) has attracted a lot of interest from researchers due to its ability to provide a game0theoretic model through which efficient allocation of frequency resource is made. The system allows for coexistence of $P$ primary (licensed) users (PUs) and $Q$ secondary (unlicensed) users (SUs) each formed by single transmitter receiver, using the same bandwidth. This bandwidth is assumed to be divided into $N$ subcarriers. The systems coexisting in the network are noncooperative and it is assumed that there is no central authority. The transmit strategy of SU $q$ is the power allocation vector $\mathbf{p}_{q}\left\{p_{q}(n)\right\}_{n=1}^{N}$ over $N$ subcarriers, subject to the constraints

$$
\hat{\mathcal{P}}_{q} \triangleq\left\{\mathbf{p} \in \mathbb{R}^{N}: \sum_{n=1}^{N} p(n) \leq P_{q}, \mathbf{0} \leq \mathbf{p} \leq \mathbf{p}_{q}^{\max }\right\}
$$

If the channel state is time varying then it cannot be assumed that perfect channel state information (CSI) is available (perfect CSI is assumed in [7] while imperfect CSI is considered in [13]). Under imperfect CSI the channel transfer function $\left\{H_{q q}\left(n, \xi_{q q}^{n}\right)\right\}_{n=1}^{N}$ and cross channel transfer function $\left\{H_{q r}\left(n, \xi_{q r}^{n}\right)\right\}_{n, r \neq q}$ can be assumed to be random variables with some known mean and bounded variance (Rayleigh fading channel assumption leads to Rayleigh distributed transfer functions). Under this setup the maximum expected information on link $q$ for a power profile $\mathbf{p}_{q}$ is given as:

$r_{q}\left(\mathbf{p}_{q}, \mathbf{p}_{-q}\right)=\sum_{n=1}^{N} \mathbb{E}\left[\log \left(1+\frac{\left|H_{q q}\left(n, \xi_{q q}^{n}\right)\right|^{2} p_{q}(n)}{\sigma_{q}^{2}(n)+\sum_{r \neq q}\left|H_{q r}\left(n, \xi_{q r}^{n}\right)\right|^{2} p_{r}(n)}\right)\right]$,

where $\mathbf{p}_{-q} \triangleq\left(\mathbf{p}_{r}\right)_{r \neq q}$. The transmission from SUs on the overlapping spectrum with the PUs causes interference resulting in degradation of quality of PU's performance. We consider the following expected value interference tolerance constraints, for each user $p=1, \ldots, P$

$$
\begin{aligned}
& \sum_{q=1}^{Q} \sum_{n=1}^{N} \mathbb{E}\left[\left|H_{p q}^{(P, S)}\left(n, \xi_{p q}^{n}\right)\right|^{2}\right] p_{q}(n) \leq P_{p, \text { tot }}^{\text {ave }} \\
& \sum_{q=1}^{Q} \mathbb{E}\left[\left|H_{p q}^{(P, S)}\left(n, \xi_{p q}^{n}\right)\right|^{2}\right] p_{q}(n) \leq P_{p, n}^{\text {peak }}, \forall n,
\end{aligned}
$$

where $H_{p q}^{P, S}\left(n, \xi_{p q}^{n}\right)$ is the channel transfer function between the transmitter of the $q$ th secondary user and the receiver of the $p$ th primary user, $P_{p, n}^{\text {peak }}$ is the maximum interference over subcarrier $n$ and $P_{p \text {,tot }}^{\text {ave }}$ is the temperature interference limit for $p$ th user. Under imperfect CSI, for any PU-SU pair $p-q$ the channel $H_{p q}^{(P, S)}\left(n, \xi_{p q}^{n}\right)$ is a random variable. 
Under this scenario, the problem faced by each secondary user $q$ is to maximize the expected information in (17) under the constraints in (16) and (18). However to deal with the global constraint, we introduce a pricing mechanism, controlled by primary users, leading to the following problem:

$$
\begin{aligned}
\max _{\mathbf{p}_{q}} & r_{q}\left(\mathbf{p}_{q}, \mathbf{p}_{-q}\right) \\
& -\sum_{p=1}^{P} \sum_{n=1}^{N}\left(\lambda_{p, n}^{\text {peak }} \mathbb{E}\left[\left|H_{p q}^{(P, S)}\left(n, \xi_{p q}^{n}\right)\right|^{2}\right] p_{q}(n)-P_{p, n}^{\text {peak }}\right) \\
& -\sum_{p=1}^{P} \lambda_{p, \text { tot }}\left(\sum_{n=1}^{N} \mathbb{E}\left[\left|H_{p q}^{(P, S)}\left(n, \xi_{p q}^{n}\right)\right|^{2}\right] p_{q}(n)-P_{p, \text { tot }}^{\text {ave }}\right)
\end{aligned}
$$$$
\text { s.t. } \mathbf{p}_{q} \in \hat{\mathcal{P}}_{q} \text {, }
$$

where $\lambda_{p}=\left(\lambda_{p, 1}^{\text {peak }}, \ldots, \lambda_{p, N}^{\text {peak }}, \lambda_{p, \text { tot }}\right)^{T}$ is the price vector of primary player $p$ for the constraints in (18) which can be compactly written as $\lambda=\left(\lambda_{1}, \ldots, \lambda_{N}\right)^{T}$. Thus the preceding can be viewed as a Lagrangian function $\mathscr{L}(\mathbf{p}, \lambda)$ defined as:

$$
\begin{aligned}
& \mathbb{E}[\mathscr{L}(\mathbf{p}, \lambda, \xi)] \triangleq\left(r_{q}\left(\mathbf{p}_{q}, \mathbf{p}_{-q}\right)\right. \\
& -\sum_{p=1}^{P} \sum_{n=1}^{N} \lambda_{p, n}^{\text {peak }}\left(\mathbb{E}\left[\left|H_{p q}^{(P, S)}\left(n, \xi_{p q}^{n}\right)\right|^{2}\right] p_{q}(n)-P_{p, n}^{\text {peak }}\right) \\
& \left.-\sum_{p=1}^{P} \lambda_{p, \text { tot }}\left(\sum_{n=1}^{N} \mathbb{E}\left[\left|H_{p q}^{(P, S)}\left(n, \xi_{p q}^{n}\right)\right|^{2}\right] p_{q}(n)-P_{p, \text { tot }}^{\text {ave }}\right)\right)_{q=1}^{Q},
\end{aligned}
$$

where $\xi: \Omega \rightarrow \mathbb{R}^{(Q+P) Q N}$ accounts for the uncertainty in various transfer functions. If we let $x=(p, \lambda)$ then a pair $x^{\mathrm{NE}}=\left(\mathbf{p}^{\mathrm{NE}}, \lambda^{\mathrm{NE}}\right)$ solves the Nash equilibrium problem if and only if $x^{\mathrm{NE}} \in \operatorname{SOL}\left(\hat{\mathcal{P}} \times \mathbb{R}_{+}^{P(N+1)}, \Phi\right)$ where $\hat{\mathcal{P}}=\hat{\mathcal{P}}_{1} \times$ $\ldots \times \hat{\mathcal{P}}_{Q}$ and

$\Phi(x)=\Phi(\mathbf{p}, \lambda) \triangleq\left(-\nabla_{\mathbf{p}} \mathbb{E}[\mathscr{L}(\mathbf{p}, \lambda, \xi)], \nabla_{\lambda} \mathbb{E}[\mathscr{L}(\mathbf{p}, \lambda, \xi)]\right)$.

In [7] the mapping $\mathbf{F}(\mathbf{p}) \triangleq\left(\mathbf{F}_{1}(\mathbf{p}), \ldots, \mathbf{F}_{Q}(\mathbf{p})^{T}\right)^{T}$ with each $\mathbf{F}_{q}(\mathbf{p})=-\nabla_{\mathbf{p}_{q}} r_{q}\left(\mathbf{p}_{q}, \mathbf{p}_{-q}\right)$ is assumed to be either strongly monotone or uniformly P-function over the set $\hat{\mathcal{P}}$ and sufficient conditions for the same are also provided. However in our case we assume that $\mathbf{F}$ is strictly monotone and further the mapping $\Phi$ is merely monotone. To this end consider the regularized mapping $\Phi_{\epsilon}$ defined as

$$
\Phi_{\epsilon} \triangleq\left(-\nabla_{\mathbf{p}} \mathbb{E}[\mathscr{L}(\mathbf{p}, \lambda, \xi)], \nabla_{\lambda} \mathbb{E}[\mathscr{L}(\mathbf{p}, \lambda, \xi)]+\epsilon \lambda\right),
$$

where $\epsilon>0$ is the regularization constant. Note that the regularized map $\Phi_{\epsilon}$ is strictly monotone which is due to the fact that mapping $\mathbf{F}$ is strictly monotone. Now we are in a position to implement the IPP in the following distributed form:

$\mathbf{p}_{q}^{k+1}=\Pi_{\hat{\mathcal{P}}_{q}}\left[\mathbf{p}_{q}^{k}-\alpha_{q}\left(-\nabla_{\mathbf{p}_{q}} \mathbb{E}[\mathscr{L}(\mathbf{p}, \lambda, \xi)]+\theta_{k}\left(\mathbf{p}_{q}^{k}-\mathbf{p}_{q}^{k-1}\right)+w_{q}^{k}\right)\right]$, $\lambda_{p}^{k+1}=\Pi_{\mathbb{R}_{+}^{N+1}}\left[\lambda_{p}^{k}-\alpha_{p}\left(\nabla_{\lambda_{p}} \mathscr{L}(\mathbf{p}, \lambda)+\epsilon \lambda_{p}^{k}+\theta_{k}\left(\lambda_{p}^{k}-\lambda_{p}^{k-1}\right)+w_{p}^{k}\right)\right]$, where $w^{k}=-\nabla \mathbb{E}[\mathscr{L}(\mathbf{p}, \lambda, \xi)]+\nabla \tilde{\mathscr{L}}\left(\mathbf{p}, \lambda, \xi^{k}\right)$, is the stochastic error vector with

$$
\nabla \tilde{\mathscr{L}}\left(\mathbf{p}, \lambda, \xi^{k}\right) \triangleq\left(\begin{array}{c}
\left.\nabla_{x_{1}} \mathscr{L}\left(\mathbf{p}, \lambda, \xi_{1}^{k}\right)\right) \\
\vdots \\
\nabla_{x_{P+Q}} \mathscr{L}\left(\mathbf{p}, \lambda, \xi_{P+Q}^{k}\right)
\end{array}\right) \text { and } \xi^{k} \triangleq\left(\begin{array}{c}
\xi_{1}^{k} \\
\vdots \\
\xi_{P+Q}^{k}
\end{array}\right) \text {. }
$$

\subsection{Numerical Results}

In this section, we discuss the application of the stochastic IPP on a CR system drawn from [13] but with modified expected value objective function and coupled constraint for SUs. The system consists of $1 \mathrm{PU}$ and 3 SUs over $N=$ 8 channels in a symmetric hexagonal topology. SU1 and 3 are subject to a common sum power budget $P_{q}=P^{\text {sum }}=$ $\infty, \mathbf{p}_{q}^{\max }=3 / 10 \mathrm{~dB}$ whereas for SU2 $P_{q}=P^{\text {sum }}=\infty$, $\mathbf{p}_{q}^{\max }=6 / 10 \mathrm{~dB}$ and a common peak interference limit $P_{p, n}^{\text {peak }}=P^{\text {peak }}=0.3 \mathrm{~dB}, P_{p, \text { tot }}^{\text {ave }}=\infty$ for all $n$ and normalized noise $\sigma_{q}^{2}(n)=1$ for all $q, n$. We assume that iid Rayleigh assumption holds and under this the distribution of channel gains $\left|H_{q r}\left(n, \xi_{q r}^{n}\right)\right|^{2}$ follows an exponential distribution with parameter $\gamma$ and $1 / \gamma=3 / 10 \mathrm{~dB}$ for $q, r, n$, where $\exp$ denotes the exponential distribution with parameter $\gamma$. The prox parameter $\theta_{k}=0.2$ for all $k$ and $\epsilon=1 \mathrm{e}-12$. The stepsize sequence follows $\alpha_{i}=\left(1000+k+\delta_{i}\right)^{-0.54}$ with $\delta_{i} \sim U(-250,250)$ where $U(a, b)$ denotes the uniform distribution over interval $(a, b)$ and $i=1, \ldots, P+Q$. We run the simulation for various values of terminating iterations $k$ for a sample size of 100 . Table 1 report the width of $90 \%$ confidence interval on the norm of the deviation from the optimal i.e. $\left\|\mathbf{p}_{q}^{k}-\mathbf{p}_{q}^{\mathrm{NE}}\right\|$ for each SU $q$ and $\left\|\lambda_{p}^{k}-\lambda_{p}^{\mathrm{NE}}\right\|$ for each PU $p$ for various $k$.

Table 1. $90 \%$ Confidence Intervals

\begin{tabular}{c|c|c|c|c}
\hline \multicolumn{5}{c}{ Width of Confidence Intervals } \\
\hline user & $k=1 \mathrm{e} 3$ & $k=1 \mathrm{e} 4$ & $k=5 \mathrm{e}$ & $k=1 \mathrm{e} 5$ \\
\hline SU 1 & $1.45 e-02$ & $1.21 e-02$ & $9.31 e-03$ & $7.53 e-03$ \\
\hline SU 2 & $1.50 e-02$ & $1.21 e-02$ & $9.31 e-03$ & $7.53 e-03$ \\
\hline SU 3 & $1.49 e-02$ & $1.21 e-02$ & $9.32 e-03$ & $7.53 e-03$ \\
\hline PU 1 & $3.23 e-02$ & $2.40 e-02$ & $1.70 e-02$ & $1.67 e-02$ \\
\hline
\end{tabular}

We also test the performance of each user by changing the distribution parameter of channel gains for SU 2 to $\gamma=10 / 6$ while SU 1 and 3 still have $\gamma=10 / 3$ for their channel gains. Note that this increase in mean also results in increased variance for an exponentially distributed channel gain function. As expected the additional randomness in the system results in increased width of the confidence intervals for all the players due to coupling through PU 1 price. The width of confidence interval is reported in Table 2.

Table 2. Effect of increasing variance

\begin{tabular}{c|c|c}
\hline \multicolumn{3}{c}{ Width of Confidence Intervals } \\
\hline user & $\gamma=10 / 3$ & $\gamma=10 / 6$ \\
\hline SU 1 & $7.53 \mathrm{e}-03$ & $7.61 \mathrm{e}-03$ \\
\hline SU 2 & $7.53 \mathrm{e}-03$ & $7.61 \mathrm{e}-03$ \\
\hline SU 3 & $7.53 \mathrm{e}-03$ & $7.61 \mathrm{e}-03$ \\
\hline PU 1 & $1.71 \mathrm{e}-02$ & $1.76 \mathrm{e}-02$ \\
\hline
\end{tabular}

While comparing our schemes to that in [7], it would be worth commenting that our schemes are gradient based in contrast to the best response schemes in [7]. Also the best 
response scheme relies on an explicit form for best response and thus difficult to implement in a stochastic regime.

\section{CONCLUDING REMARKS}

This paper is motivated by the need to compute equilibria arising in cognitive radio systems in uncertain regimes. Specifically, such systems lead to stochastic Nash games in which the mappings do not display a desirable strong monotonicity property. Past work by Jiang and Xu [4] considered how stochastic approximation procedures can address stochastic variational inequalities when the mappings were strongly monotone. Yet, these schemes cannot easily contend with weaker requirements (such as strict monotonicity) while retaining the single-timescale structure. Instead, a simple regularization-based extension leads to a two timescale method, that is generally harder to implement in networked settings.

Accordingly, in this paper we develop a single-timescale proximal point methods in which the centering term is updated at every iterate and provide almost-sure convergence of the resulting scheme. We analyze this method under common and different steplengths across players and implement the algorithm on a Nash equilibrium problem arising in the design of cognitive radio with temperature interference constraints when the players utility have strictly monotone gradient maps.

\section{REFERENCES}

[1] M. Bloem, T. Alpcan, and T. Başar, A stackelberg game for power control and channel allocation in cognitive radio networks, ValueTools '07: Proceedings of the 2nd international conference on Performance evaluation methodologies and tools (ICST, Brussels, Belgium, Belgium), ICST (Institute for Computer Sciences, Social-Informatics and Telecommunications Engineering), 2007, pp. 1-9.

[2] Y. Ermoliev, Stochastic programming methods, Nauka, Moscow, 1976.

[3] F. Facchinei and J.-S. Pang, Finite-Dimensional Variational Inequalities and Complementarity Problems, vol. 1 and 2, Springer-Verlag Inc., New York, 2003.

[4] H. Jiang and H. Xu, Stochastic approximation approaches to the stochastic variational inequality problem, IEEE Transactions on Automatic Control, 53 (2008), no. 6, 1462-1475.

[5] P-H. Lin, S-C. Lin, and H-J. Su, Cognitive radio with partial channel state information at the transmitter, IEEE International Conference on Communications, 2008, pp. 1065-1071.
[6] B. Martinet, Régularisation d'inéquations variationnelles par approximations successives, Rev. Française Informat. Recherche Opérationnelle 4 (1970), no. Ser. R-3, 154-158. MR 0298899 (45 \#7948)

[7] J-S. Pang, G. Scutari, D.P. Palomar, and F. Facchinei, Design of cognitive radio systems under TemperatureInterference constraints: A variational inequality approach, IEEE Transactions on Signal Processing, 58 (2010), no. 6, 3251-3271.

[8] B.T. Polyak, Introduction to optimisation, Optimization Software, Inc., New York, 1987.

[9] H. Robbins and S. Monro, A stochastic approximation method, The Annals of Mathematical Statistics 22 (1951), no. 3, 400-407.

[10] R.T. Rockafellar, Augmented lagrangians and applications of the proximal point algorithm in convex programming, Mathematics of Operations Research 1 (1976), no. $2,97-116$.

[11] A.N. Tikhonov, On the solution of incorrectly put problems and the regularisation method, Outlines Joint Sympos. Partial Differential Equations (Novosibirsk, 1963), Acad. Sci. USSR Siberian Branch, Moscow, 1963, pp. 261-265. MR MR0211218 (35 \#2100)

[12] J. Wang and D.P. Palomar, Worst-Case robust MIMO transmission with imperfect channel knowledge, IEEE Transactions on Signal Processing, 57 (2009), no. 8, 3086-3100.

[13] J. Wang, G. Scutari, and D.P. Palomar, Robust cognitive radio via game theory, 2010 IEEE International Symposium on Information Theory Proceedings (ISIT), 2010, pp. 2073-2077. 